Commun. Fac. Sci. Univ. Ank. Ser. A1 Math. Stat.

Volume 68, Number 2, Pages 1909-1921(2019)

DOI: $10.31801 /$ cfsuasmas. 505287

ISSN 1303-5991 E-ISSN 2618-6470

http://communications.science.ankara.edu.tr/index.php?series=A1

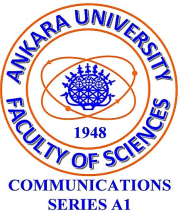

\title{
CERTAIN SUBCLASSES OF BI-UNIVALENT FUNCTIONS RELATED TO $k$-FIBONACCI NUMBERS
}

\author{
H. ÖZLEM GÜNEY, G. MURUGUSUNDARAMOORTHY, AND J. SOKÓŁ
}

\begin{abstract}
In this paper, we introduce and investigate new subclasses of biunivalent functions related to $k$-Fibonacci numbers. Furthermore, we find estimates of first two coefficients of functions in these classes. Also, we obtain the Fekete-Szegö inequalities for these function classes.
\end{abstract}

\section{INTRODUCTION}

Let $\mathbb{D}=\{z:|z|<1\}$ be the unit disc in the complex plane. The class of all analytic functions

$$
f(z)=z+\sum_{n=2}^{\infty} a_{n} z^{n}
$$

in the open unit disc $\mathbb{D}$ with normalization $f(0)=0, f^{\prime}(0)=1$ is denoted by $\mathcal{A}$ and the class $\mathcal{S} \subset \mathcal{A}$ is the class which consists of univalent functions in $\mathbb{D}$. We say that $f$ is subordinate to $F$ in $\mathbb{D}$, written as $f \prec F$, if and only if $f(z)=F(\omega(z))$ for some analytic function $\omega,|\omega(z)| \leq|z|, z \in \mathbb{D}$.

The Koebe one quarter theorem [5] ensures that the image of $\mathbb{D}$ under every univalent function $f \in \mathcal{A}$ contains a disk of radius $1 / 4$. Thus every univalent function $f$ has an inverse $f^{-1}$ satisfying

$$
f^{-1}(f(z))=z,(z \in \mathbb{D}) \text { and } f\left(f^{-1}(w)\right)=w,\left(|w|<r_{0}(f), r_{0}(f) \geq \frac{1}{4}\right) .
$$

A function $f \in \mathcal{A}$ is said to be bi-univalent in $\mathbb{D}$ if $f$ is univalent in $\mathbb{D}$ and $f^{-1}$ has an univalent extension to $\mathbb{D}$. Let $\Sigma$ denote the class of bi-univalent functions defined in the unit disk $\mathbb{D}$. Someone can see a short history and examples of functions in the class $\Sigma$ in [14. Since $f \in \Sigma$ has the Maclaurin series given by (1), a computation shows that its inverse $g=f^{-1}$ has the expansion

$$
g(w)=f^{-1}(w)=w-a_{2} w^{2}+\left(2 a_{2}^{2}-a_{3}\right) w^{3}+\cdots .
$$

Received by the editors: December 30, 2018; Accepted: January 14, 2019.

2010 Mathematics Subject Classification. Primary 30C45; Secondary 30C50.

Key words and phrases. Analytic functions, bi-univalent, $k$-Fibonacci numbers, starlike functions, convex functions.

(C)2019 Ankara University Communications Faculty of Sciences University of Ankara-Series A1 Mathematics and Statistics 
The work of Srivastava et al. [14 essentially revived the investigation of various subclasses of the bi-univalent function class in recent years. In a considerably large number of sequels to the aforementioned work of Srivastava et al. [14, several different subclasses of the bi-univalent function class $\Sigma$ were introduced and studied analogously by many authors (see, for example, 1, 2, 4, 8, 3, 15, 9, ), but only nonsharp estimates on the initial coefficients $\left|a_{2}\right|$ and $\left|a_{3}\right|$ in the Taylor-Maclaurin expansion (1) were obtained in these recent papers.

The object of the present work is to introduce a new subclass of the function class $\Sigma$ and find estimates on the coefficients $\left|a_{2}\right|$ and $\left|a_{3}\right|$ for functions in this new subclass of the function class $\Sigma$ using the technique of Srivastava et al. 14

Recently,Yilmaz Özgür and Sokół 10 introduced the class $\mathcal{S} \mathcal{L}^{k}$ of starlike functions connected with $k$-Fibonacci numbers as the set of functions $f \in \mathcal{A}$ which is described in the following definition.

Definition 1. Let $k$ be any positive real number. The function $f \in \mathcal{A}$ belongs to the class $\mathcal{S L}^{k}$ if it satisfies the condition that

$$
\frac{z f^{\prime}(z)}{f(z)} \prec \widetilde{p}_{k}(z), \quad z \in \mathbb{D},
$$

where

$$
\widetilde{p}_{k}(z)=\frac{1+\tau_{k}^{2} z^{2}}{1-k \tau_{k} z-\tau_{k}^{2} z^{2}}, \tau_{k}=\frac{k-\sqrt{k^{2}+4}}{2}, \quad z \in \mathbb{D} .
$$

Later in [7, Güney et al. defined the class $\mathcal{K} \mathcal{S} \mathcal{L}^{k}$ as follows:

Definition 2. Let $k$ be any positive real number. The function $f \in \mathcal{A}$ belongs to the class $\mathcal{K S}^{k}{ }^{k}$ if it satisfies the condition that

$$
1+\frac{z f^{\prime \prime}(z)}{f^{\prime}(z)} \prec \widetilde{p}_{k}(z), \quad z \in \mathbb{D},
$$

where the function $\widetilde{p}_{k}$ is defined in (3).

For $k=1$, the classes $\mathcal{S} \mathcal{L}$ and $\mathcal{K S} \mathcal{L}$ of shell-like functions were defined in [12] (see also [13]).

It was proved in [10] that functions in the class $\mathcal{S} \mathcal{L}^{k}$ are univalent in $\mathbb{D}$. Moreover, the class $\mathcal{S} \mathcal{L}^{k}$ is a subclass of the class of starlike functions $\mathcal{S}^{*}$, even more, starlike of order $k\left(k^{2}+4\right)^{-1 / 2} / 2$. The name attributed to the class $\mathcal{S} \mathcal{L}^{k}$ is motivated by the shape of the curve

$$
\mathcal{C}=\left\{\widetilde{p}_{k}\left(e^{i t}\right): t \in[0,2 \pi) \backslash\{\pi\}\right\} .
$$

Now we define the classes $\mathcal{S} \mathcal{L} \mathcal{M}_{\alpha}^{k}$ and $\mathcal{S} \mathcal{L} \mathcal{G}_{\gamma}^{k}$, as follows:

Definition 3. Let $k$ be any positive real number. The function $f \in \mathcal{A}$ belongs to the class $\mathcal{S L M}_{\alpha}^{k},(0 \leq \alpha \leq 1)$ if it satisfies the condition that

$$
\alpha\left(1+\frac{z f^{\prime \prime}(z)}{f^{\prime}(z)}\right)+(1-\alpha) \frac{z f^{\prime}(z)}{f(z)} \prec \widetilde{p}_{k}(z), \quad z \in \mathbb{D},
$$


where the function $\widetilde{p}_{k}$ is defined in (3).

Definition 4. Let $0 \leq \gamma \leq 1$, and $k$ be any positive real number. The function $f \in A$ belongs to the class $S L G_{\gamma}^{k}$ if the following conditions are satisfied:

$$
\left(\frac{z f^{\prime}(z)}{f(z)}\right)^{\gamma}\left(1+\frac{z f^{\prime \prime}(z)}{f^{\prime}(z)}\right)^{1-\gamma} \prec \tilde{p}_{k}(z), \quad z \in \mathbb{D},
$$

where the function $\widetilde{p}_{k}$ is defined in (3).

For $k \leq 2$, note that we have

$$
\widetilde{p}_{k}\left(e^{ \pm i \arccos \left(k^{2} / 4\right)}\right)=k\left(k^{2}+4\right)^{-1 / 2},
$$

and so the curve $\mathcal{C}$ intersects itself on the real axis at the point $w_{1}=k\left(k^{2}+4\right)^{-1 / 2}$. Thus $\mathcal{C}$ has a loop intersecting the real axis also at the point $w_{2}=\left(k^{2}+4\right) /(2 k)$. For $k>2$, the curve $\mathcal{C}$ has no loops and it is like a conchoid, see for details [10]. Moreover, the coefficients of $\widetilde{p}_{k}$ are connected with $k$-Fibonacci numbers.

For any positive real number $k$, the $k$-Fibonacci number sequence $\left\{F_{k, n}\right\}_{n=0}^{\infty}$ is defined recursively by

$$
F_{k, 0}=0, F_{k, 1}=1 \text { and } F_{k, n+1}=k F_{k, n}+F_{k, n-1} \text { for } n \geq 1 .
$$

When $k=1$, we obtain the well-known Fibonacci numbers $F_{n}$. It is known that the $n^{t h} k$-Fibonacci number is given by

$$
F_{k, n}=\frac{\left(k-\tau_{k}\right)^{n}-\tau_{k}^{n}}{\sqrt{k^{2}+4}},
$$

where $\tau_{k}=\left(k-\sqrt{k^{2}+4}\right) / 2$. If $\widetilde{p}_{k}(z)=1+\sum_{n=1}^{\infty} \widetilde{p}_{k, n} z^{n}$, then we have

$$
\widetilde{p}_{k, n}=\left(F_{k, n-1}+F_{k, n+1}\right) \tau_{k}^{n}, n=1,2,3, \ldots
$$

Also, Özgür and Sokół showed in [10] that

$$
\begin{aligned}
\widetilde{p}_{k}(z)=\frac{1+\tau_{k}^{2} z^{2}}{1-k \tau_{k} z-\tau_{k}^{2} z^{2}} & =1+\sum_{n=1}^{\infty} \widetilde{p}_{k, n} z^{n} \\
& =1+\left(F_{k, 0}+F_{k, 2}\right) \tau_{k} z+\left(F_{k, 1}+F_{k, 3}\right) \tau_{k}^{2} z^{2}+\cdots \\
& =1+k \tau_{k} z+\left(k^{2}+2\right) \tau_{k}^{2} z^{2}+\left(k^{3}+3 k\right) \tau_{k}^{3} z^{3}+\cdots .
\end{aligned}
$$

where $\tau_{k}=\frac{k-\sqrt{k^{2}+4}}{2}, \quad z \in \mathbb{D}$, (see [10]).

Let $\mathcal{P}(\beta), 0 \leq \beta<1$, denote the class of analytic functions $p$ in $\mathbb{D}$ with $p(0)=1$ and $\operatorname{Re}\{p(z)\}>\beta$. Especially, we use $\mathcal{P}(0)=\mathcal{P}$ as $\beta=0$.

Now we give the following lemma which will use in proving.

Lemma 5. ([11]) Let $p \in \mathcal{P}$ with $p(z)=1+c_{1} z+c_{2} z^{2}+\cdots$, then

$$
\left|c_{n}\right| \leq 2 \quad \text { for } \quad n \geq 1 .
$$




\section{Bi-Univalent FUnCtion CLASS $\mathcal{S} \mathcal{L} \mathcal{M}_{\alpha, \Sigma}^{k}\left(\widetilde{p}_{k}(z)\right)$}

In this section, we introduce three new subclasses of $\Sigma$ associated with shell-like functions connected with Fibonacci numbers and obtain the initial Taylor coefficients $\left|a_{2}\right|$ and $\left|a_{3}\right|$ for the function classes by subordination.

Firstly, let $p(z)=1+p_{1} z+p_{2} z^{2}+\cdots$, and $p \prec \tilde{p}_{k}$. Then there exists an analytic function $u$ such that $|u(z)|<1$ in $\mathbb{U}$ and $p(z)=\tilde{p}_{k}(u(z))$. Therefore, the function

$$
h(z)=\frac{1+u(z)}{1-u(z)}=1+c_{1} z+c_{2} z^{2}+\ldots
$$

is in the class $\mathcal{P}(0)$. It follows that

$$
u(z)=\frac{c_{1} z}{2}+\left(c_{2}-\frac{c_{1}^{2}}{2}\right) \frac{z^{2}}{2}+\left(c_{3}-c_{1} c_{2}+\frac{c_{1}^{3}}{4}\right) \frac{z^{3}}{2}+\cdots
$$

and

$$
\begin{aligned}
\tilde{p}_{k}(u(z))= & 1+\tilde{p}_{k, 1}\left\{\frac{c_{1} z}{2}+\left(c_{2}-\frac{c_{1}^{2}}{2}\right) \frac{z^{2}}{2}+\left(c_{3}-c_{1} c_{2}+\frac{c_{1}^{3}}{4}\right) \frac{z^{3}}{2}+\cdots\right\} \\
& +\tilde{p}_{k, 2}\left\{\frac{c_{1} z}{2}+\left(c_{2}-\frac{c_{1}^{2}}{2}\right) \frac{z^{2}}{2}+\left(c_{3}-c_{1} c_{2}+\frac{c_{1}^{3}}{4}\right) \frac{z^{3}}{2}+\cdots\right\}^{2} \\
& +\tilde{p}_{k, 3}\left\{\frac{c_{1} z}{2}+\left(c_{2}-\frac{c_{1}^{2}}{2}\right) \frac{z^{2}}{2}+\left(c_{3}-c_{1} c_{2}+\frac{c_{1}^{3}}{4}\right) \frac{z^{3}}{2}+\cdots\right\}^{3}+\cdots \\
& =1+\frac{\tilde{p}_{k, 1} c_{1} z}{2}+\left\{\frac{1}{2}\left(c_{2}-\frac{c_{1}^{2}}{2}\right) \tilde{p}_{k, 1}+\frac{c_{1}^{2}}{4} \tilde{p}_{k, 2}\right\} z^{2} \\
& +\left\{\frac{1}{2}\left(c_{3}-c_{1} c_{2}+\frac{c_{1}^{3}}{4}\right) \tilde{p}_{k, 1}+\frac{1}{2} c_{1}\left(c_{2}-\frac{c_{1}^{2}}{2}\right) \tilde{p}_{k, 2}+\frac{c_{1}^{3}}{8} \tilde{p}_{k, 3}\right\} z^{3}+\cdots .
\end{aligned}
$$

And similarly, there exists an analytic function $v$ such that $|v(w)|<1$ in $\mathbb{D}$ and $p(w)=\tilde{p}_{k}(v(w))$. Therefore, the function

$$
k(w)=\frac{1+v(w)}{1-v(w)}=1+d_{1} w+d_{2} w^{2}+\ldots
$$

is in the class $\mathcal{P}(0)$. It follows that

$$
v(w)=\frac{d_{1} w}{2}+\left(d_{2}-\frac{d_{1}^{2}}{2}\right) \frac{w^{2}}{2}+\left(d_{3}-d_{1} d_{2}+\frac{d_{1}^{3}}{4}\right) \frac{w^{3}}{2}+\cdots
$$

and

$$
\begin{aligned}
\tilde{p}_{k}(v(w)) & =1+\frac{\tilde{p}_{k, 1} d_{1} w}{2}+\left\{\frac{1}{2}\left(d_{2}-\frac{d_{1}^{2}}{2}\right) \tilde{p}_{k, 1}+\frac{d_{1}^{2}}{4} \tilde{p}_{k, 2}\right\} w^{2} \\
& +\left\{\frac{1}{2}\left(d_{3}-d_{1} d_{2}+\frac{d_{1}^{3}}{4}\right) \tilde{p}_{k, 1}+\frac{1}{2} d_{1}\left(d_{2}-\frac{d_{1}^{2}}{2}\right) \tilde{p}_{k, 2}+\frac{d_{1}^{3}}{8} \tilde{p}_{k, 3}\right\} w^{3}+\cdots .
\end{aligned}
$$


Definition 6. For $0 \leq \alpha \leq 1$, a function $f \in \Sigma$ of the form (1) is said to be in the class $\mathcal{S} \mathcal{L} \mathcal{M}_{\alpha, \Sigma}^{k}\left(\widetilde{p}_{k}(z)\right)$ if the following subordination hold:

$$
\alpha\left(1+\frac{z f^{\prime \prime}(z)}{f^{\prime}(z)}\right)+(1-\alpha)\left(\frac{z f^{\prime}(z)}{f(z)}\right) \prec \widetilde{p}_{k}(z)=\frac{1+\tau_{k}^{2} z^{2}}{1-k \tau_{k} z-\tau_{k}^{2} z^{2}},
$$

and

$$
\alpha\left(1+\frac{w g^{\prime \prime}(w)}{g^{\prime}(w)}\right)+(1-\alpha)\left(\frac{w g^{\prime}(w)}{g(w)}\right) \prec \widetilde{p}_{k}(w)=\frac{1+\tau_{k}^{2} w^{2}}{1-k \tau_{k} w-\tau_{k}^{2} w^{2}},
$$

where $\tau_{k}=\frac{k-\sqrt{k^{2}+4}}{2}$ where $z, w \in \mathbb{D}$ and $g$ is given by (2).

Specializing the parameter $\alpha=0$ and $\alpha=1$ we have the following:

Definition 7. A function $f \in \Sigma$ of the form (1) is said to be in the class $\mathcal{S L}_{\Sigma}^{k}\left(\tilde{p}_{k}(z)\right)$ if the following subordination hold:

$$
\frac{z f^{\prime}(z)}{f(z)} \prec \widetilde{p}_{k}(z)=\frac{1+\tau_{k}^{2} z^{2}}{1-k \tau_{k} z-\tau_{k}^{2} z^{2}},
$$

and

$$
\frac{w g^{\prime}(w)}{g(w)} \prec \widetilde{p}_{k}(w)=\frac{1+\tau_{k}^{2} w^{2}}{1-k \tau_{k} w-\tau_{k}^{2} w^{2}},
$$

where $\tau_{k}=\frac{k-\sqrt{k^{2}+4}}{2}, z, w \in \mathbb{D}$ and $g$ is given by (2).

Definition 8. A function $f \in \Sigma$ of the form (1) is said to be in the class $\mathcal{K} \mathcal{S} \mathcal{L}_{\Sigma}^{k}\left(\tilde{p}_{k}(z)\right)$ if the following subordination hold:

$$
1+\frac{z f^{\prime \prime}(z)}{f^{\prime}(z)} \prec \widetilde{p}_{k}(z)=\frac{1+\tau_{k}^{2} z^{2}}{1-k \tau_{k} z-\tau_{k}^{2} z^{2}},
$$

and

$$
1+\frac{w g^{\prime \prime}(w)}{g^{\prime}(w)} \prec \widetilde{p}_{k}(w)=\frac{1+\tau_{k}^{2} w^{2}}{1-k \tau_{k} w-\tau_{k}^{2} w^{2}},
$$

where $\tau_{k}=\frac{k-\sqrt{k^{2}+4}}{2}, z, w \in \mathbb{D}$ and $g$ is given by (2).

In the following theorem we determine the initial Taylor coefficients $\left|a_{2}\right|$ and $\left|a_{3}\right|$ for the function class $\mathcal{S} \mathcal{L} \mathcal{M}_{\alpha, \Sigma}^{k}\left(\widetilde{p}_{k}(z)\right)$. Later we state the bounds to other classes as a special cases.

Theorem 9. Let $f$ given by (1) be in the class $\mathcal{S} \mathcal{L} \mathcal{M}_{\alpha, \Sigma}^{k}\left(\widetilde{p}_{k}(z)\right)$. Then

$$
\left|a_{2}\right| \leq \frac{k \sqrt{k}\left|\tau_{k}\right|}{\sqrt{(1+\alpha)^{2} k-(1+\alpha)\left(2(1+\alpha)+\alpha k^{2}\right) \tau_{k}}}
$$

and

$$
\left|a_{3}\right| \leq \frac{k\left|\tau_{k}\right|\left\{(1+\alpha)^{2} k-\left[\left(k^{2}+2\right) \alpha^{2}+\left(5 k^{2}+4\right) \alpha+2\left(k^{2}+1\right)\right] \tau_{k}\right\}}{2(1+2 \alpha)(1+\alpha)\left[(1+\alpha) k-\left(2(1+\alpha)+\alpha k^{2}\right) \tau_{k}\right]} .
$$


Proof. Let $f \in \mathcal{S} \mathcal{L} \mathcal{M}_{\alpha, \Sigma}^{k}\left(\widetilde{p}_{k}(z)\right)$ and $g=f^{-1}$. Considering (11) and (12), we have

$$
\alpha\left(1+\frac{z f^{\prime \prime}(z)}{f^{\prime}(z)}\right)+(1-\alpha)\left(\frac{z f^{\prime}(z)}{f(z)}\right)=\tilde{p_{k}}(u(z))
$$

and

$$
\alpha\left(1+\frac{w g^{\prime \prime}(w)}{g^{\prime}(w)}\right)+(1-\alpha)\left(\frac{w g^{\prime}(w)}{g(w)}\right)=\tilde{p_{k}}(v(w)),
$$

where $\tau_{k}=\frac{k-\sqrt{k^{2}+4}}{2}, z, w \in \mathbb{D}$ and $g$ is given by (2). We have also

$$
\begin{aligned}
& \alpha\left(1+\frac{z f^{\prime \prime}(z)}{f^{\prime}(z)}\right)+(1-\alpha)\left(\frac{z f^{\prime}(z)}{f(z)}\right) \\
= & 1+(1+\alpha) a_{2} z+\left(2(1+2 \alpha) a_{3}-(1+3 \alpha) a_{2}^{2}\right) z^{2}+\ldots \\
= & 1+\frac{\tilde{p}_{k, 1} c_{1} z}{2}+\left[\frac{1}{2}\left(c_{2}-\frac{c_{1}^{2}}{2}\right) \tilde{p}_{k, 1}+\frac{c_{1}^{2}}{4} \tilde{p}_{k, 2}\right] z^{2} \\
& +\left[\frac{1}{2}\left(c_{3}-c_{1} c_{2}+\frac{c_{1}^{3}}{4}\right) \tilde{p}_{k, 1}+\frac{1}{2} c_{1}\left(c_{2}-\frac{c_{1}^{2}}{2}\right) \tilde{p}_{k, 2}+\frac{c_{1}^{3}}{8} \tilde{p}_{k, 3}\right] z^{3}+\cdots
\end{aligned}
$$

and

$$
\begin{aligned}
& \alpha\left(1+\frac{w g^{\prime \prime}(w)}{g^{\prime}(w)}\right)+(1-\alpha)\left(\frac{w g^{\prime}(w)}{g(w)}\right) \\
= & 1-(1+\alpha) a_{2} w+\left((3+5 \alpha) a_{2}^{2}-2(1+2 \alpha) a_{3}\right) w^{2}+\ldots \\
= & 1+\frac{\tilde{p}_{k, 1} d_{1} w}{2}+\left[\frac{1}{2}\left(d_{2}-\frac{d_{1}^{2}}{2}\right) \tilde{p}_{k, 1}+\frac{d_{1}^{2}}{4} \tilde{p}_{k, 2}\right] w^{2} \\
+ & {\left[\frac{1}{2}\left(d_{3}-d_{1} d_{2}+\frac{d_{1}^{3}}{4}\right) \tilde{p}_{k, 1}+\frac{1}{2} d_{1}\left(d_{2}-\frac{d_{1}^{2}}{2}\right) \tilde{p}_{k, 2}+\frac{d_{1}^{3}}{8} \tilde{p}_{k, 3}\right] w^{3}+\cdots }
\end{aligned}
$$

It follows from 21 and 22 that

$$
\begin{gathered}
(1+\alpha) a_{2}=\frac{c_{1} k \tau_{k}}{2}, \\
2(1+2 \alpha) a_{3}-(1+3 \alpha) a_{2}^{2}=\frac{1}{2}\left(c_{2}-\frac{c_{1}^{2}}{2}\right) k \tau_{k}+\frac{c_{1}^{2}}{4}\left(k^{2}+2\right) \tau_{k}^{2},
\end{gathered}
$$

and

$$
\begin{gathered}
-(1+\alpha) a_{2}=\frac{d_{1} k \tau_{k}}{2}, \\
(3+5 \alpha) a_{2}^{2}-2(1+2 \alpha) a_{3}=\frac{1}{2}\left(d_{2}-\frac{d_{1}^{2}}{2}\right) k \tau_{k}+\frac{d_{1}^{2}}{4}\left(k^{2}+2\right) \tau_{k}^{2} .
\end{gathered}
$$

From (23) and 25), we have

$$
c_{1}=-d_{1},
$$

and

$$
2 a_{2}^{2}=\frac{\left(c_{1}^{2}+d_{1}^{2}\right)}{4(1+\alpha)^{2}} k^{2} \tau_{k}^{2}
$$


Now, by summing (24) and (26), we obtain

$$
2(1+\alpha) a_{2}^{2}=\frac{1}{2}\left(c_{2}+d_{2}\right) k \tau_{k}-\frac{1}{4}\left(c_{1}^{2}+d_{1}^{2}\right) k \tau_{k}+\frac{1}{4}\left(c_{1}^{2}+d_{1}^{2}\right)\left(k^{2}+2\right) \tau_{k}^{2} .
$$

By putting (28) in 29), we have

$$
2(1+\alpha)\left[\left(-2(1+\alpha)-\alpha k^{2}\right) \tau_{k}+(1+\alpha) k\right] a_{2}^{2}=\frac{1}{2}\left(c_{2}+d_{2}\right) k^{3} \tau_{k}^{2} .
$$

Therefore, using Lemma 5 we obtain

$$
\left|a_{2}\right| \leq \frac{k \sqrt{k}\left|\tau_{k}\right|}{\sqrt{(1+\alpha)^{2} k-(1+\alpha)\left(2(1+\alpha)+\alpha k^{2}\right) \tau_{k}}} .
$$

Now, so as to find the bound on $\left|a_{3}\right|$, let's subtract from (24) and (26). So, we find

$$
4(1+2 \alpha) a_{3}-4(1+2 \alpha) a_{2}^{2}=\frac{1}{2}\left(c_{2}-d_{2}\right) k \tau_{k} .
$$

Hence, we get

$$
4(1+2 \alpha)\left|a_{3}\right| \leq 2 k\left|\tau_{k}\right|+4(1+2 \alpha)\left|a_{2}\right|^{2} .
$$

Then, in view of (31), we obtain

$$
\left|a_{3}\right| \leq \frac{k\left|\tau_{k}\right|\left\{(1+\alpha)^{2} k-\left[2(1+\alpha)^{2}+\left(\alpha^{2}+5 \alpha+2\right) k^{2}\right] \tau_{k}\right\}}{2(1+2 \alpha)(1+\alpha)\left[(1+\alpha) k-\left(2(1+\alpha)+\alpha k^{2}\right) \tau_{k}\right]} .
$$

If we can take the parameter $\alpha=0$ and $\alpha=1$ in the above theorem, we have the following the initial Taylor coefficients $\left|a_{2}\right|$ and $\left|a_{3}\right|$ for the function classes $\mathcal{S} \mathcal{L}_{\Sigma}^{k}\left(\tilde{p}_{k}(z)\right)$ and $\mathcal{K} \mathcal{S} \mathcal{L}_{\Sigma}^{k}\left(\tilde{p}_{k}(z)\right)$, respectively.

Corollary 10. Let $f$ given by (1) be in the class $\mathcal{S L}_{\Sigma}^{k}\left(\tilde{p}_{k}(z)\right)$. Then

$$
\left|a_{2}\right| \leq \frac{k \sqrt{k}\left|\tau_{k}\right|}{\sqrt{k-2 \tau_{k}}}
$$

and

$$
\left|a_{3}\right| \leq \frac{k\left|\tau_{k}\right|\left\{k-2\left(k^{2}+1\right) \tau_{k}\right\}}{2\left(k-2 \tau_{k}\right)} .
$$

Corollary 11. Let $f$ given by (1) be in the class $\mathcal{K S}_{\mathcal{L}}^{k}\left(\tilde{p}_{k}(z)\right)$. Then

$$
\left|a_{2}\right| \leq \frac{k \sqrt{k}\left|\tau_{k}\right|}{\sqrt{4 k-2\left(4+k^{2}\right) \tau_{k}}}
$$

and

$$
\left|a_{3}\right| \leq \frac{k\left|\tau_{k}\right|\left\{k-2\left(k^{2}+1\right) \tau_{k}\right\}}{3\left(2 k-\left(4+k^{2}\right) \tau_{k}\right)} .
$$


If we can take the parameter $k=1$ in the above corollaries, we have the following the initial Taylor coefficients $\left|a_{2}\right|$ and $\left|a_{3}\right|$ for the function classes $\mathcal{S} \mathcal{L}_{\Sigma}(\tilde{p}(z))$ and $\mathcal{K} \mathcal{S} \mathcal{L}_{\Sigma}(\tilde{p}(z))$, respectively, which were obtained in [6] by Güney et.al.

Corollary 12. Let $f$ given by (1) be in the class $\mathcal{S} \mathcal{L}_{\Sigma}(\tilde{p}(z))$. Then

$$
\left|a_{2}\right| \leq \frac{|\tau|}{\sqrt{1-2 \tau}}
$$

and

$$
\left|a_{3}\right| \leq \frac{|\tau|(1-4 \tau)}{2(1-2 \tau)}
$$

Corollary 13. Let $f$ given by (1) be in the class $\mathcal{K} \mathcal{S L}_{\Sigma}(\tilde{p}(z))$. Then

$$
\left|a_{2}\right| \leq \frac{|\tau|}{\sqrt{4-10 \tau}}
$$

and

$$
\left|a_{3}\right| \leq \frac{|\tau|(1-4 \tau)}{3(2-5 \tau)}
$$

\section{Bi-Univalent FUnCtion Class $\mathcal{S} \mathcal{L} \mathcal{G}_{\gamma, \Sigma}^{k}\left(\widetilde{p}_{k}(z)\right)$}

In this section, we define a new class $\mathcal{S} \mathcal{L} \mathcal{G}_{\gamma, \Sigma}^{k}\left(\widetilde{p}_{k}(z)\right)$ of $\gamma$ - bi-starlike functions associated with shell-like domain.

Definition 14. Let $0 \leq \gamma \leq 1$, and $k$ be any positive real number. A function $f \in \Sigma$ of the form (1) is said to be in the class $S L G_{\gamma, \Sigma}^{k}\left(\widetilde{p}_{k}(z)\right)$ if the following subordination hold:

$$
\left(\frac{z f^{\prime}(z)}{f(z)}\right)^{\gamma}\left(1+\frac{z f^{\prime \prime}(z)}{f^{\prime}(z)}\right)^{1-\gamma} \prec \tilde{p}_{k}(z)
$$

and

$$
\left(\frac{w g^{\prime}(w)}{g(w)}\right)^{\gamma}\left(1+\frac{w g^{\prime \prime}(w)}{g^{\prime}(w)}\right)^{1-\gamma} \prec \tilde{p}_{k}(w),
$$

where the function $\widetilde{p}_{k}$ is defined in (3) and $z, w \in D$.

Remark 15. Taking $\gamma=1$, we get $\mathcal{S} \mathcal{L} \mathcal{G}_{1, \Sigma}^{k}\left(\widetilde{p}_{k}(z)\right) \equiv \mathcal{S L}_{\Sigma}^{k}\left(\tilde{p}_{k}(z)\right)$ the class as given in Definition 7 satisfying the conditions given in (13) and (14).

Remark 16. Taking $\gamma=0$, we get $\mathcal{S} \mathcal{L} \mathcal{G}_{0, \Sigma}^{k}\left(\widetilde{p}_{k}(z)\right) \equiv \mathcal{K} \mathcal{S} \mathcal{L}_{\Sigma}^{k}\left(\tilde{p}_{k}(z)\right)$ the class as given in Definition 8 satisfying the conditions given in (15) and (16).

Theorem 17. Let $f$ given by (1) be in the class $S L G_{\gamma, \Sigma}^{k}\left(\widetilde{p}_{k}(z)\right)$. Then

$$
\left|a_{2}\right| \leq \frac{k \sqrt{2 k}\left|\tau_{k}\right|}{\sqrt{2(2-\gamma)^{2} k-\left(4(2-\gamma)^{2}+\left(\gamma^{2}-5 \gamma+4\right) k^{2}\right) \tau_{k}}}
$$


and

$$
\left|a_{3}\right| \leq \frac{k\left|\tau_{k}\right|\left[2(2-\gamma)^{2} k-\left(4(2-\gamma)^{2}+\left(\gamma^{2}-13 \gamma+16\right) k^{2}\right) \tau_{k}\right]}{2(3-2 \gamma)\left[2 k(2-\gamma)^{2}-\left(4(2-\gamma)^{2}+\left(\gamma^{2}-5 \gamma+4\right) k^{2}\right) \tau_{k}\right]}
$$

Proof. Let $f \in \mathcal{S} \mathcal{L} \mathcal{G}_{\gamma, \Sigma}^{k}\left(\widetilde{p}_{k}(z)\right)$ and $g=f^{-1}$ given by (2). Considering (1) and (2), we have

and

$$
\left(\frac{z f^{\prime}(z)}{f(z)}\right)^{\gamma}\left(1+\frac{z f^{\prime \prime}(z)}{f^{\prime}(z)}\right)^{1-\gamma}=\tilde{p}_{k}(u(z))
$$

$$
\left(\frac{w g^{\prime}(w)}{g(w)}\right)^{\gamma}\left(1+\frac{w g^{\prime \prime}(w)}{g^{\prime}(w)}\right)^{1-\gamma}=\tilde{p}_{k}(v(w))
$$

where the function $\widetilde{p}_{k}$ is defined in $(3), z, w \in \mathbb{D}$ and $g$ is given by (2). We also have

$$
\begin{aligned}
& \left(\frac{z f^{\prime}(z)}{f(z)}\right)^{\gamma}\left(1+\frac{z f^{\prime \prime}(z)}{f^{\prime}(z)}\right)^{1-\gamma} \\
= & 1+(2-\gamma) a_{2} z+\left(2(3-2 \gamma) a_{3}+\frac{1}{2}\left[(\gamma-2)^{2}-3(4-3 \gamma)\right] a_{2}^{2}\right) z^{2}+\ldots
\end{aligned}
$$

and

$$
\begin{aligned}
& \left(\frac{w g^{\prime}(w)}{g(w)}\right)^{\gamma}\left(1+\frac{w g^{\prime \prime}(w)}{g^{\prime}(w)}\right)^{1-\gamma} \\
= & 1-(2-\gamma) a_{2} w+\left(\left[8(1-\gamma)+\frac{1}{2} \gamma(\gamma+5)\right] a_{2}^{2}-2(3-2 \gamma) a_{3}\right) w^{2}+\ldots
\end{aligned}
$$

Equating the coefficients in (5) and (6), with (7)-(10), respectively, we get,

$$
\begin{aligned}
(2-\gamma) a_{2} & =\frac{c_{1} k \tau_{k}}{2} \\
2(3-2 \gamma) a_{3}+\frac{1}{2}\left[(\gamma-2)^{2}-3(4-3 \gamma)\right] a_{2}^{2} & =\frac{1}{2}\left(c_{2}-\frac{c_{1}^{2}}{2}\right) k \tau_{k}+\frac{c_{1}^{2}}{4}\left(k^{2}+2\right) \tau_{k}^{2},
\end{aligned}
$$

and

$$
\begin{aligned}
-(2-\gamma) a_{2} & =\frac{d_{1} k \tau_{k}}{2} \\
-2(3-2 \gamma) a_{3}+\left[8(1-\gamma)+\frac{1}{2} \gamma(\gamma+5)\right] a_{2}^{2} & =\frac{1}{2}\left(d_{2}-\frac{d_{1}^{2}}{2}\right) k \tau_{k}+\frac{d_{1}^{2}}{4}\left(k^{2}+2\right) \tau_{k}^{2}
\end{aligned}
$$

From $(7)$ and $(9)$, we have

$$
a_{2}=\frac{c_{1} k \tau_{k}}{2(2-\gamma)}=-\frac{d_{1} k \tau_{k}}{2(2-\gamma)}
$$

which implies

$$
c_{1}=-d_{1}
$$


and

$$
a_{2}^{2}=\frac{\left(c_{1}^{2}+d_{1}^{2}\right) k^{2} \tau_{k}^{2}}{8(2-\gamma)^{2}}
$$

Now, by summing $(8)$ and $(10)$, we obtain

$$
\left(\gamma^{2}-3 \gamma+4\right) a_{2}^{2}=\frac{1}{2}\left(c_{2}+d_{2}\right) k \tau_{k}-\frac{1}{4}\left(c_{1}^{2}+d_{1}^{2}\right) k \tau_{k}+\frac{1}{4}\left(c_{1}^{2}+d_{1}^{2}\right)\left(k^{2}+2\right) \tau_{k}^{2} .
$$

Proceeding similarly as in the earlier proof of Theorem 9 and using Lemma 5 we obtain

$$
\left|a_{2}\right| \leq \frac{k \sqrt{2 k}\left|\tau_{k}\right|}{\sqrt{2(2-\gamma)^{2} k-\left(4(2-\gamma)^{2}+\left(\gamma^{2}-5 \gamma+4\right) k^{2}\right) \tau_{k}}} .
$$

Now, so as to find the bound on $\left|a_{3}\right|$, let's subtract from $(8)$ and $(10)$. So, we find

$$
4(3-2 \gamma) a_{3}-4(3-2 \gamma) a_{2}^{2}=\frac{1}{2}\left(c_{2}-d_{2}\right) k \tau_{k} .
$$

Hence, we get

$$
4(3-2 \gamma)\left|a_{3}\right| \leq 2 k\left|\tau_{k}\right|+4(3-2 \gamma)\left|a_{2}\right|^{2} .
$$

Then, in view of (11), we obtain

$$
\left|a_{3}\right| \leq \frac{k\left|\tau_{k}\right|\left[2(2-\gamma)^{2} k-\left(4(2-\gamma)^{2}+\left(\gamma^{2}-13 \gamma+16\right) k^{2}\right) \tau_{k}\right]}{2(3-2 \gamma)\left[2 k(2-\gamma)^{2}-\left(4(2-\gamma)^{2}+\left(\gamma^{2}-5 \gamma+4\right) k^{2}\right) \tau_{k}\right]} .
$$

Remark 18. By taking $\gamma=1$ and $\gamma=0$ in the above theorem, we have the initial Taylor coefficients $\left|a_{2}\right|$ and $\left|a_{3}\right|$ for the function classes $\mathcal{S L}_{\Sigma}^{k}\left(\tilde{p}_{k}(z)\right)$ and $\mathcal{K} \mathcal{S} \mathcal{L}_{\Sigma}^{k}\left(\tilde{p}_{k}(z)\right)$, as stated in Corollary 10 and Corollary 11 respectively. Further note that by taking $k=1$ we have the initial Taylor coefficients $\left|a_{2}\right|$ and $\left|a_{3}\right|$ for the function classes $\mathcal{S L}_{\Sigma}(\tilde{p}(z))$ and $\mathcal{K} \mathcal{S} \mathcal{L}_{\Sigma}(\tilde{p}(z))$, as stated in Corollary 12 and Corollary 13 respectively.

\section{Fekete-Szegö inequalities for the above function Classes}

Due to Zaprawa [16, we will give Fekete-Szegö inequalities for the above function classes in this section. The first theorem is the solution of the Fekete-Szegö problem in $\mathcal{S} \mathcal{L} \mathcal{M}_{\alpha, \Sigma}^{k}\left(\widetilde{p}_{k}(z)\right)$ and it looks like the following:

Theorem 19. Let $f$ given by (1) be in the class $\mathcal{S L M}_{\alpha, \Sigma}^{k}\left(\widetilde{p}_{k}(z)\right)$ and $\mu \in \mathbb{R}$. Then we have

$$
\left|a_{3}-\mu a_{2}^{2}\right| \leq \begin{cases}\frac{k\left|\tau_{k}\right|}{2(1+2 \alpha)}, & |\mu-1| \leq \frac{4(1+\alpha)\left[(1+\alpha) k-\left(2(1+\alpha)+\alpha k^{2}\right) \tau_{k}\right]}{8(1+2 \alpha) k^{2}\left|\tau_{k}\right|}, \\ \frac{|1-\mu| k^{3} \tau_{k}^{2}}{(1+\alpha)\left[(1+\alpha) k-\left(2(1+\alpha)+\alpha k^{2}\right) \tau_{k}\right]}, & |\mu-1| \geq \frac{4(1+\alpha)\left[(1+\alpha) k-\left(2(1+\alpha)+\alpha k^{2}\right) \tau_{k}\right]}{8(1+2 \alpha) k^{2}\left|\tau_{k}\right|}\end{cases}
$$


Proof. From 30 and 32 we obtain

$$
\begin{gathered}
a_{3}-\mu a_{2}^{2}=(1-\mu) \frac{k^{3} \tau_{k}^{2}\left(c_{2}+d_{2}\right)}{4(1+\alpha)\left[(1+\alpha) k-\left(2(1+\alpha)+\alpha k^{2}\right) \tau_{k}\right]}+\frac{k \tau_{k}\left(c_{2}-d_{2}\right)}{8(1+2 \alpha)} \\
=\left(\frac{(1-\mu) k^{3} \tau_{k}^{2}}{4(1+\alpha)\left[(1+\alpha) k-\left(2(1+\alpha)+\alpha k^{2}\right) \tau_{k}\right]}+\frac{k \tau_{k}}{8(1+2 \alpha)}\right) c_{2} \\
+\left(\frac{(1-\mu) k^{3} \tau_{k}^{2}}{4(1+\alpha)\left[(1+\alpha) k-\left(2(1+\alpha)+\alpha k^{2}\right) \tau_{k}\right]}-\frac{k \tau_{k}}{8(1+2 \alpha)}\right) d_{2} .
\end{gathered}
$$

So we have

$$
a_{3}-\mu a_{2}^{2}=\left(h(\mu)-\frac{k\left|\tau_{k}\right|}{8(1+2 \alpha)}\right) c_{2}+\left(h(\mu)+\frac{k\left|\tau_{k}\right|}{8(1+2 \alpha)}\right) d_{2}
$$

where

$$
h(\mu)=\frac{(1-\mu) k^{3} \tau_{k}^{2}}{4(1+\alpha)\left[(1+\alpha) k-\left(2(1+\alpha)+\alpha k^{2}\right) \tau_{k}\right]} .
$$

Then, by taking modulus of (2), we conclude that

$$
\left|a_{3}-\mu a_{2}^{2}\right| \leq \begin{cases}\frac{k\left|\tau_{k}\right|}{2(1+2 \alpha)}, & 0 \leq|h(\mu)| \leq \frac{k\left|\tau_{k}\right|}{8(1+2 \alpha)} \\ 4|h(\mu)|, & |h(\mu)| \geq \frac{k\left|\tau_{k}\right|}{8(1+2 \alpha)}\end{cases}
$$

Taking $\mu=1$, we have the following corollary.

Corollary 20. If $f \in \mathcal{S} \mathcal{L} \mathcal{M}_{\alpha, \Sigma}^{k}\left(\widetilde{p}_{k}(z)\right)$, then

$$
\left|a_{3}-a_{2}^{2}\right| \leq \frac{k\left|\tau_{k}\right|}{2(1+2 \alpha)} .
$$

The second theorem is the solution of the Fekete-Szegö problem in $\mathcal{S} \mathcal{L G}_{\gamma, \Sigma}^{k}\left(\widetilde{p}_{k}(z)\right)$ and it looks like the following:

Theorem 21. Let $f$ given by (1) be in the class $\mathcal{S} \mathcal{L} \mathcal{G}_{\gamma, \Sigma}^{k}\left(\widetilde{p}_{k}(z)\right)$ and $\mu \in \mathbb{R}$. Then we have

$$
\left|a_{3}-\mu a_{2}^{2}\right| \leq \begin{cases}\frac{k\left|\tau_{k}\right|}{2(3-2 \gamma)}, & |\mu-1| \leq \frac{2(2-\gamma)^{2} k-\left(4(2-\gamma)^{2}+\left(\gamma^{2}-5 \gamma+4\right) k^{2}\right) \tau_{k}}{4(3-2 \gamma) k^{2}\left|\tau_{k}\right|}, \\ \frac{2|1-\mu| k^{3} \tau_{k}^{2}}{2(2-\gamma)^{2} k-\left(4(2-\gamma)^{2}+\left(\gamma^{2}-5 \gamma+4\right) k^{2}\right) \tau_{k}}, & |\mu-1| \geq \frac{2(2-\gamma)^{2} k-\left(4(2-\gamma)^{2}+\left(\gamma^{2}-5 \gamma+4\right) k^{2}\right) \tau_{k}}{4(3-2 \gamma) k^{2}\left|\tau_{k}\right|} .\end{cases}
$$

Taking $\mu=1$, we have the following corollary.

Corollary 22. If $f \in \mathcal{S L G}_{\gamma, \Sigma}^{k}\left(\widetilde{p}_{k}(z)\right)$, then

$$
\left|a_{3}-a_{2}^{2}\right| \leq \frac{k\left|\tau_{k}\right|}{2(3-2 \gamma)} .
$$


If we can take the parameter $\alpha=0$ and $\alpha=1$ in the Theorem 19 or $\gamma=1$ and $\gamma=0$ in the Theorem 21, we have the following the Fekete-Szegö inequalities for the function classes $\mathcal{S} \mathcal{L}_{\Sigma}^{k}\left(\tilde{p}_{k}(z)\right)$ and $\mathcal{K} \mathcal{S L}_{\Sigma}^{k}\left(\tilde{p}_{k}(z)\right)$, respectively.

Corollary 23. Let $f$ given by (1) be in the class $\mathcal{S} \mathcal{L}_{\Sigma}^{k}\left(\tilde{p}_{k}(z)\right)$ and $\mu \in \mathbb{R}$. Then we have

$$
\left|a_{3}-\mu a_{2}^{2}\right| \leq \begin{cases}\frac{k\left|\tau_{k}\right|}{2}, & |\mu-1| \leq \frac{k-2 \tau_{k}}{2 k^{2}\left|\tau_{k}\right|}, \\ \frac{|1-\mu| k^{3} \tau_{k}^{2}}{k-2 \tau_{k}}, & |\mu-1| \geq \frac{k-2 \tau_{k}}{2 k^{2}\left|\tau_{k}\right|} .\end{cases}
$$

Corollary 24. Let $f$ given by (1) be in the class $\mathcal{K} \mathcal{S L}_{\Sigma}^{k}\left(\tilde{p}_{k}(z)\right)$ and $\mu \in \mathbb{R}$. Then we have

$$
\left|a_{3}-\mu a_{2}^{2}\right| \leq \begin{cases}\frac{k\left|\tau_{k}\right|}{6}, & |\mu-1| \leq \frac{2 k-\left(k^{2}+4\right) \tau_{k}}{3 k^{2}\left|\tau_{k}\right|}, \\ \frac{|1-\mu| k^{3} \tau_{k}^{2}}{2\left(2 k-\left(k^{2}+4\right) \tau_{k}\right)}, & |\mu-1| \geq \frac{2 k-\left(k^{2}+4\right) \tau_{k}}{3 k^{2}\left|\tau_{k}\right|} .\end{cases}
$$

\section{Concluding Remarks and Observations}

In our present investigation, we have introduced new classes $\mathcal{S} \mathcal{L} \mathcal{M}_{\alpha, \Sigma}^{k}\left(\widetilde{p}_{k}(z)\right)$ and $\mathcal{S} \mathcal{L G}_{\gamma, \Sigma}^{k}\left(\widetilde{p}_{k}(z)\right)$ of bi-univalent functions in the open unit disk U. For the initial Taylor- Maclaurin coefficients of functions belonging to these classes, we have studied the problem of finding the upper bound associated with the Fekete-Szegö inequality. We have also considered several results which are closely related to our investigation in this paper.

\section{ACKNOWLEDGEMENT}

The authors would like to thank the referees for the helpful suggestions.

\section{REFERENCES}

[1] Brannan D.A., Clunie J. and Kirwan W.E., Coefficient estimates for a class of star-like functions, Canad. J. Math., Vol.22 (1970), 476-485.

[2] Brannan D.A. and Taha T.S., On some classes of bi-univalent functions, Studia Univ. BabesBolyai Math., Vol. 31, No.2 (1986), 70-77.

[3] Bulut S., Certain subclasses of analytic and bi-univalent functions involving the q-derivative operator, Commun. Fac. Sci. Univ. Ank. SÃ@)r. A1 Math. Stat., Vol.66 (2017), 108-114.

[4] Çağlar M. and Deniz E., Initial coefficients for a subclass of bi-univalent functions defined by Salagean differential operator, Commun. Fac. Sci. Univ. Ank. SÃ@r. A1 Math. Stat., Vol.66 (2017), 85-91.

[5] Duren P.L., Univalent Functions, in: Grundlehren der Mathematischen Wissenschaften, Band 259, New York, Berlin, Heidelberg and Tokyo, Springer-Verlag, 1983.

[6] Güney H.Ö., Murugusundaramoorthy G. and Sokół J., Subclasses of bi-univalent functions related to shell-like curves connected with Fibonacci numbers, Acta Univers. Sapientiae, Mathematica, Vol.10, No.1 (2018), 70-84.

[7] Güney H.Ö., Sokół J. and İlhan S., Second Hankel determinant problem for some analytic function classes connected with $k$-Fibonacci numbers, Acta Univers. Apulensis, Vol.54 (2018), $161-174$. 
[8] Lewin M., On a coefficient problem for bi-univalent functions, Proc. Amer. Math. Soc., Vol.18 (1967), 63-68.

[9] Li X-F. and Wang A-P., Two new subclasses of bi-univalent functions, Inter. Math. Forum, Vol.7, No.30 (2012), 1495-1504.

[10] Özgür N.Y. and Sokół J., On starlike functions connected with $k$-Fibonacci numbers, Bull. Malaysian Math. Sci. Soc., Vol.38, No.1 (2015), 249-258.

[11] Pommerenke, Ch., Univalent Functions, in: Studia Mathematica Mathematische Lehrbucher, Vanderhoeck and Ruprecht, Göttingen, 1975.

[12] Sokół J., On starlike functions connected with Fibonacci numbers, Folia Scient. Univ. Tech. Resoviensis, Vol.175, No.23 (1999), 111-116.

[13] Sokół J., Remarks on shell-like functions, Folia Scient. Univ. Tech. Resoviensis, Vol.181, No.24 (2000), 111-115.

[14] Srivastava H.M., Mishra A.K. and Gochhayat P., Certain subclasses of analytic and biunivalent functions, Appl. Math. Lett., Vol.23, No.10 (2010), 1188-1192.

[15] Xu Q.-H., Gui Y.-C. and Srivastava H.M., Coefficinet estimates for a certain subclass of analytic and bi-univalent functions, Appl. Math. Lett., Vol.25 (2012), 990-994.

[16] Zaprawa P., On the Fekete-Szegö problem for classes of bi-univalent functions, Bull. Belg. Math. Soc. Simon Stevin, Vol.21, No.1 (2014), 169-178.

Current address: H. Özlem Güney: Dicle University, Faculty of Science, Department of Mathematics, Diyarbakır- Turkey.

E-mail address: ozlemg@dicle.edu.tr

ORCID Address: http://orcid.org/0000-0002-3010-7795

Current address: G. Murugusundaramoorthy: School of Advanced Sciences, VIT University, Vellore -632014, India.

E-mail address: gmsmoorthy@yahoo.com

ORCID Address: http://orcid.org/0000-0001-8285-6619

Current address: J. Sokół: University of Rzeszów, Faculty of Mathematics and Natural Sciences, ul. Prof. Pigonia 1, 35-310 Rzeszów, Poland.

E-mail address: jsokol@ur.edu.pl

ORCID Address: http://orcid.org/0000-0003-1204-2286 